\title{
Large-B-Cell Lymphoma causing Intussusception in a Young Male: A Challenge for a General Hospital.
}

\author{
D. Cussa ${ }^{1}, \mathrm{~S}$. Mantova $^{1}$, A. Fornari ${ }^{2}, \mathrm{~S}$. Reddavid $^{1}$, M. Degiuli ${ }^{1}$ \\ ${ }^{1}$ Department of Surgical Oncology and Digestive Surgery, ${ }^{2}$ Department of Pathology; University of Turin, A.O.U. San Luigi \\ Gonzaga Orbassano (Turin), Italy.
}

\section{Corresponding Author: \\ Dr. Davide Cussa}

Email id: davide.cussa@hotmail.it

This is an Open Access article distributed under the terms of the Creative Commons Attribution License (creativecommons.org/ licenses/by/3.0).

Received Accepted

Published

July 17,2019

September 5, 2019

September 15, 2019

\begin{abstract}
Background: Intussusception is a rare cause of bowel obstruction in adults. Even if intussusception in children is almost always a benign condition due to a congenital laxity of mesentery; in young adults this condition can be often a consequence of a malignancy like an appendiceal neuroendocrine tumor or lymphoma. Case Report: We present a case of bowel intussusception in a 16 years old male admitted to our Emergency Department for recurrent abdominal pain, who underwent a right hemicolectomy after CT scan and colonoscopy. Pathological examination showed a large B-cell lymphoma of the appendiceal foramen leading to a colo-colonic intussusception with bowel obstruction. Conclusion: Intussusception in young adults must be investigated further as it may be symptom of a malignancy.
\end{abstract}

Keywords: Abdominal Pain, Colectormy, Intestinal Obstruction, Intussusception, Lymphoma.

\section{Introduction}

Intussuception is a common condition in children, especially in those aged between 4 and 5 years old, due to a congenital laxity of mesentery, and often represents a cause of intestinal obstruction. It is called primary idiopathic intussusception in children and it is mostly treated by air enema reduction [1].

Intussusception in adults represents less than $5 \%$ [2] of cases of intestinal obstruction, but in contrast to pediatric population a detectable primary cause can be found in more than $70 \%$ of cases. Approximately $40 \%$ of these nonidiopathic intussusception are caused by primary or secondary malignant neoplasms [3]. In literature less than 40 case reports have described lymphoma as the primary cause of intestinal obstruction due to intussusception in both adults and children. In more than $90 \%$ of these cases pathological examination documented the presence of a B-Cell non Hodgkin's lymphoma (NHL) [4]. The clinical presentation of this rare entity in adults often unclear and different from presentation in children where an acute abdomen, with palpable mass, is often associated to vomits and bloody mucoid stools. On the opposite, in young adults the initial diagnosis is often miss. We report our experience of a 16 year-old young male, admitted to our emergency department for recurrent abdominal pain, who underwent a right hemicolectomy due to a NHL colic lesion causing colo-colonic intussusception with bowel obstruction.

\section{Case Report}

A 16-year-old young male presented to our emergency department with a four weeks history of recurrent abdominal pain. He reported a rather non-specific progressively severe low abdominal pain. He had not experienced associated fever 
or vomiting. Unremarkable medical history was reported before this episode. In emergency room blood pressure was 120 over $80 \mathrm{mmHg}$, and heart rate was 50 beats per minute. Physical examination revealed diffuse tenderness of the abdomen, with a palpable mass in the right hypocondrium. Laboratory data, including C-reactive protein (20 $\mathrm{mg} / \mathrm{L})$ and white blood cell count $\left(8.17 \times 10^{3} \mu / \mathrm{L}\right)$ were almost normal.

Considering these clinical and laboratory findings, an abdominal ultrasound evaluation was requested. This examination showed a suspected colo-colonic intussusception of the distal right colon with mild peri-visceral fluid associated to reactive lymph-nodes findings [Fig.1-2]. Consequently to these observations, contrast enhanced abdominal CT-scan was performed. The computed tomograms revealed bowel obstruction and confirmed colocolonic intussusception at the hepatic flexure, with wall thickening. Few adjacent inflammatory nodes were also observed [Fig.3]. In view of patient's stable conditions, an emergency colonoscopy was performed to better investigate the cause of the intussusception. Furthermore, particularly in childhood, this manouvre can convert into an operative procedure of non-invasive reduction; however, the colonoscopy was interrupted, due to the high risk of perforation, when a necrotic intra-luminal lesion causing bowel intussusception was clearly diagnosed. Considering these clinical findings emergency surgery was soon scheduled. Because of the endoscopic findings, due to the fragility of the involved bowel, an open approach was choosen. The intussusception previously described by imaging was confirmed at intraoperative palpation, an intra-luminal lesion suspected as appendiceal mass was appreciated and several nodes, suspected as inflammatory or reactive were detected in the right mesocolon. According to these findings a right hemicolectomy was performed following oncological benchmarks.

The patient was discharged on seventh postoperative day, with an uneventful post-operative

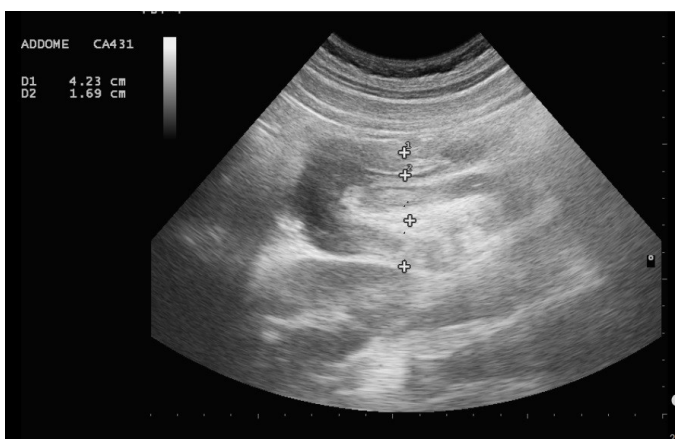

Fig.1: Ultrasonography: right colon tract (about $17 \mathrm{~mm}$ ) invaginated in hypoecogen colic tract (global involved colon about $43 \mathrm{~mm}$ ).

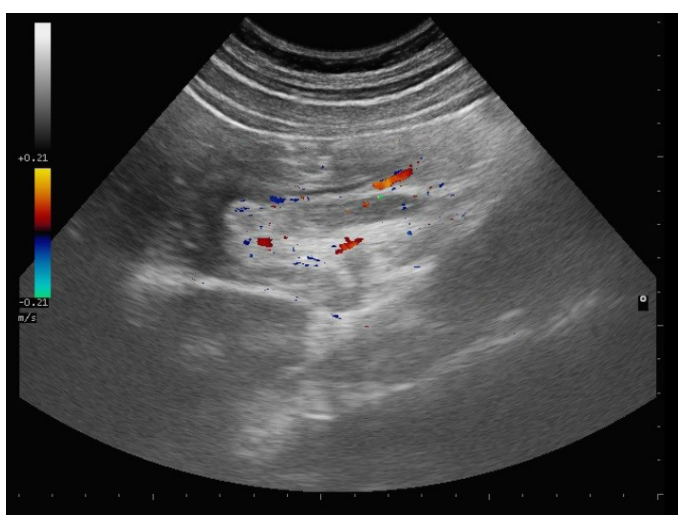

Fig.2: Ultrasonography: Typical "doughnut sign", suspicious for intussusception.

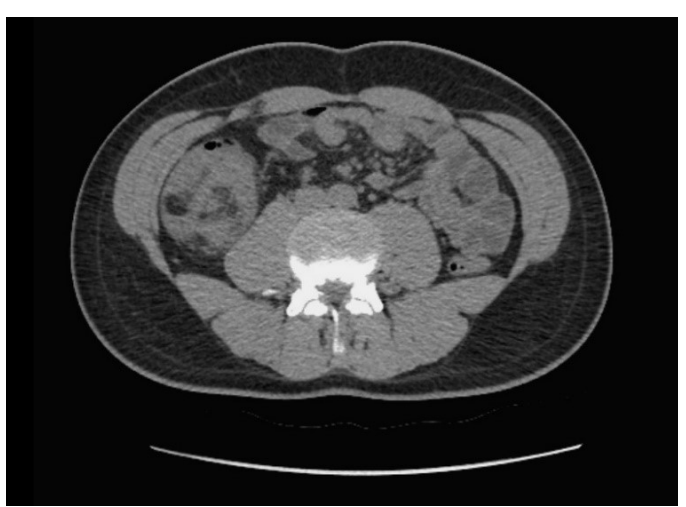

Fig.3: CT scan: confirmed the "donought sign", some lymph nodes suspicious for inflammatory reactions are detected.

course. A neoplastic mass tissue of about $5 \mathrm{~cm}$ invaginating toward the cecum and originating from the appendiceal foramen was stained for pathological examination. At gross examination an intraluminal mass lesion originating from the appendiceal foramen was seen, measuring $5 \times 5 \mathrm{~cm}$, 
and showing several areas of necrosis. Microscopy analysis of the lesion confirmed mucosal necrosis. Immunohistochemistry showed nuclear positivity for CD20, CD45 and Bcl-6 along with negativity for Bcl-2, MUM-1, and CD5. Ki-67 proliferation index value was $>90 \%$ [Fig.4-5]. Therefore, the final histological diagnosis was primary large-Bcell lymphoma of the cecum with 27 peri-visceral inflammatory lymph nodes.

\section{Discussion}

Bowel intussusception in adults represents an uncommon disease and very often develops secondary to a malignancy [5]. Moreover, in young patients the complete diagnosis of this clinical condition can be very challenging, especially in a General Hospital and for General Surgeons who are called on to choose the best treatment and to face an oncological disease in emergency setting. In fact a small bowel lymphoma should be always considered in young patients (above 5 years) with presenting symptoms of intussusception [6]. Recent study reports show this potentially dramatic disease confusing different clinical presentations. Ultrasound alone cannot define a clear clinical condition, can be very useful to demonstrate some specific disease-related imagings, like the characteristic "donut sign", as reported in this clinical case description [Fig.3]. CT scan can confirm this specific sign [9], showing with greater accuracy the site of intussusception, whether small bowel, cecum or colon. Intravenous contrast injection can improve the diagnosis of lymphadenopathy close to intussusception, differentiating inflammatory from metastatic nodes.

The risk of bowel perforation and its availability in emergency are the main limitations of colonoscopy in primary and secondary care hospitals. Anyway, when performed, it can give a direct vision of the obstruction and allows immediate bites of the pathological mass causing intussusception. In the present clinical case, emergency colonoscopy showed a necrotic area

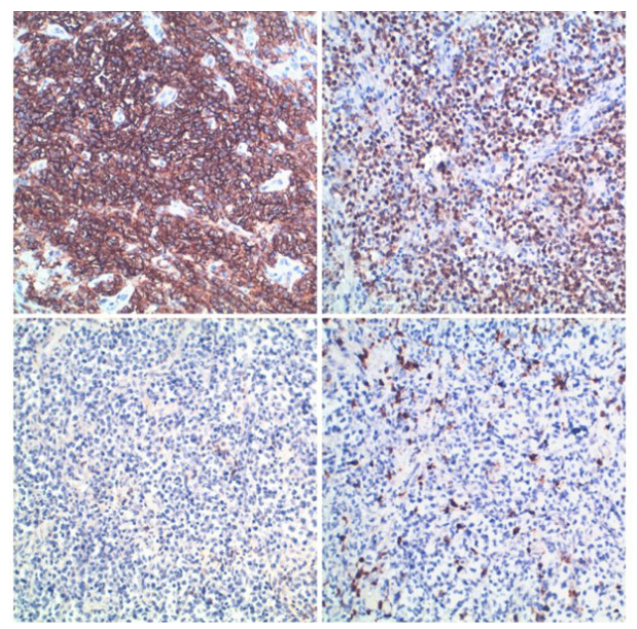

Fig.4: Immunohistochemistry showed an intense and diffuse staining for CD20 and BCL6, while BCL2 and CD5 markers were negative on neoplastic cells.

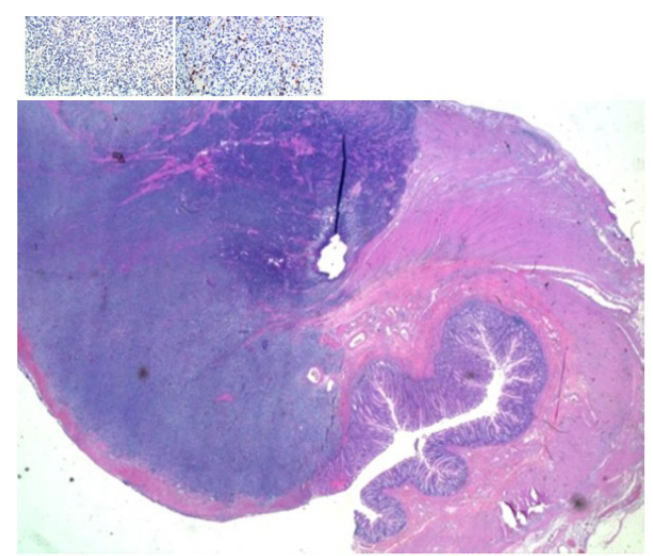

Fig.5: This low-power magnification image (H\&E staining) shows a neoplastic mass infiltrating the intestinal wall from the mucosa (ulcerated) to the serosal surface.

inside the intussuscepted cecum and therefore it was soon stopped in favour of surgery due to the high risk of bowel perforation during endoscopic manouvres. Even in case of uncertain diagnosis, several reports in literature recommend a right hemicolectomy for ileocecal intussussception, together with loco-regional lymph node dissection. The possibility that an unknown malignancy is the real cause of the intussusception should always be considered. Therefore, particularly in the adults, in whom a bowel cancer is often associated, the indication to regional lymph node dissection is mandatory. On the opposite, in childhood, the 
presence of a lymphoma is often observed and therefore in these cases surgery should be followed by chemotherapy. In the present case, a Burkitt's lymphoma (BL), which is a highly malignant and rapidly growing B-cell neoplasm [1] was histologically diagnosed.

$\mathrm{BL}$ is a highly aggressive disease invading bone marrow in $35 \%$ of cases and central nervous system in $15 \%$ of patients. Three clinical variants are described: endemic, sporadic and immunodeficiency-related subtypes. The endemic BL is linked to the Ebstein Barr virus (EBV); it is almost exclusively encountered in paediatric population of Equatorial Africa and affects kidneys and facial bones, especially jaws. Sporadic and immunodeficiency-related variants are the most common BLs in Western Europe and United States. BL onset is often related to human immunodeficiency virus (HIV) and, less frequently, to immunomodulatory treatment following transplantation or to congenital primary immunodeficiency diseases. Sporadic BL (sBL) accounts for $1-2 \%$ of lymphomas in adults and upto $40 \%$ of lymphomas in children; it is characterized by abdominal involvement and the most common affected sites are terminal ileum, cecum and intra-abdominal lymph nodes. Abdominal pain, bowel obstruction, gastrointestinal bleeding or intussusception can occur at its presentation [11-14].
For diagnosis, surgical excision/incisional biopsy of lymph nodes or extranodal pathological tissue is required to obtain an adequate tumor sample, which should be evaluated by skilled pathologists in terms of morphology, cellular phenotype and molecular expression. According to actual recommendations, adequate immunophenotyping with immunochemistry panel (CD45, CD20, CD3, CD10, Ki-67, BCL2, BCL6, TdT), with or without cell surface markers analysis (kappa/lambda, CD45, CD20, CD3, CD5, CD19, CD10, TdT) and karyotype analysis for MYC rearrangement are essential to establish the proper diagnosis [14]. For accurate staging of the disease, contrast enhanced CT scan of the neck, chest, abdomen and pelvis is mandatory. Magnetic resonance imaging (MRI) or CT scan of brain, orbits and sinuses is required in case of suspected involvement of central nervous system or craniofacial area. Positron emission tomography (PET) is recommended to improve the staging accuracy, especially in case of extra-nodal disease. The stage is defined according to the Ann Arbor classification system or St. Jude/Murphy staging system as described in Table 1 [14].

Intensive and short duration regimens of chemotherapy such as CODOX-M or $\mathrm{EPOCH}$ regimen are the most effective in $\mathrm{BL}$ treatment. CODOX-M is a combination of cyclophosphamide, vincristine, doxorubicin, intrathecal methotrexate and cytarabine, followed

Table 1: St. Jude (or Murphy) and Ann Arbor classification system for Burkitt Lymphoma staging.

\begin{tabular}{|l|l|l|l|}
\hline Stage & St. Jude (Murphy) & Stage & Ann Arbour \\
\hline I & $\begin{array}{l}\text { Single nodal or extranodal site excluding } \\
\text { mediastinum and abdomen. }\end{array}$ & I & $\begin{array}{l}\text { Involvement of a single lymphatic region or localized } \\
\text { involvement of single extralymphatic organ or site. }\end{array}$ \\
\hline II & Two or more nodal areas on one side of diaphragm. & II & $\begin{array}{l}\text { Involvement of two or more lymphatic regions on the same } \\
\text { side of the diaphragm or localized involvement of a single } \\
\text { extralymphatic organ or site and of one or more lymphatic } \\
\text { regions on the same side of the diaphragm. }\end{array}$ \\
\cline { 1 - 2 } IIR & $\begin{array}{l}\text { Completely resected intra-abdominal disease. } \\
\text { III }\end{array}$ & $\begin{array}{l}\text { Two or more nodal areas on opposite side of } \\
\text { unresectable intra-abdominal disease or paraspinal } \\
\text { or epidural tumor. }\end{array}$ & $\begin{array}{l}\text { Involvement of lymphatic regions on both sides of the } \\
\text { diaphragm. }\end{array}$ \\
\hline IV & $\begin{array}{l}\text { Involvement of bone marrow or central nervous } \\
\text { system. }\end{array}$ & IV & $\begin{array}{l}\text { Diffuse or disseminated involvement of one or mor extra } \\
\text { lymphatic organs with or without lymphatic involvement. }\end{array}$ \\
\hline
\end{tabular}


by high-dose systemic methotrexate. This schedule is alternately administered with IVAC (Ifosfamide, Etoposide, high-dose cytarabine) in case of highrisk disease. EPOCH regimen consists of the association of etoposide, prednisone, vincristine, cyclophosphamide and doxorubicin. Rituximab, a humanized monoclonal antibody against CD20 can be administered in combination with chemotherapy to improve the response $[11,12,14]$.

Survival rate after administration of chemotherapy and rituximab is more than $80 \%$. Old age, low performance status, high blood LDH level, bone marrow or central nervous system involvement is correlated with a worse prognosis [11].

\section{Conclusion}

Intussusception in young adults and adolescents could be challenge for surgeons in a General Hopspital. Furhet investigations and extended resection are needed as it could be an uncommon presentation of malignancies.

Contributors: DC: manuscript writing; SM: reviewing the literature and manuscript writing. AF: interpreting the histopathologic studies of this article and manuscript editing. SR: reviewing the literature; MD: manuscript editing. DC will act as a study guarantor. All authors approved the final version of this manuscript.

Funding: None; Competing interests: None stated.

\section{References}

1. Kulendran K, Choy KT, Keogh C, Ratnapala D. An exceptional case of ileocolic intussusception secondary to Burkitt's lymphoma: What variations are there in the presentation and management of those patients who approach adolescence? Case Rep Surg. 2018;2018:1-5.

2. Wang SM, Huang FC, Wu CH, Ko SF, Lee SY, Hsiao CC. Ileocecal Burkitt's lymphoma presenting as ileocolic intussusception with appendiceal invagination and acute appendicitis. J Formos Med Assoc. 2010;109:476-479.
3. Jayabackthan L, Kini R, Murgi S, Graham S. A rare case of primary lymphoma of the caecum presenting as intussusception. J Lab Physicians. 2013;5(2):118.

4. Akbulut S. Unusual cause of adult intussusception: Diffuse large B-cell non-Hodgkin's lymphoma: A case report and review. European Review for Medical and Pharmacological Sciences. 2012;16:1938-1946.

5. Kaval S, Singhal BM, Kumar S, Singh CP. Adult intussusception: An institutional experience and review of literature. Arch Int Surg. 2014;4(1):25-30.

6. England RJ, Pillay K, Davidson A, Numanoglu A, Millar AJW. Intussusception as a presenting feature of Burkitt lymphoma: Implications for management and outcome. Pediatr Surg Int. 2012;28:267-270.

7. Hanan B, Diniz TR, da Luz MM, da Conceição SA, da Silva RG, Lacerda-Filho A. Intussusception in adults: a retrospective study. Colorectal Dis. 2010;12:574-578.

8. Batista BN, Maximiano LF. Intestinal intussusception in young adults: literature review. Rev Col Bras Cir. 2009;36(6):533-536.

9. Belhamidi MS, Kaoukabi A, Krimou H, Menfaa M, Sakit $\mathrm{F}$, Choho K. Lymphome colique révélé par invagination iléo-caecale chez l'adulte: à propos d'un cas. Pan Afr Med J [Internet]. 2018;30:105.

10. Özant A, Arslan K, Özçay N, Besim H. Adult multicentric burkitt lymphoma with bowel obstruction due to intussusception. Turk J Gastroenterol. 2018;29(3):361364.

11. Tilly H, Gomes da Silva M, Vitolo U, Jack A, Meignan M, Lopez-Guillermo A, et al. Diffuse large B-cell lymphoma (DLBCL): ESMO Clinical Practice Guidelines for diagnosis, treatment and follow-up. Ann Oncol. 2015;26 Supple 5:v116-125.

12. Bonnet C, Janssens A, Wu KL, Schroyens W, Van Hende $\mathrm{V}$, Heimann $\mathrm{P}$, et al. BHS guidelines for the treatment of Burkitt lymphoma. Belgian J Hematol. 2015;6(2):61-69.

13. Blum KA, Lozanski G, Byrd JC. Adult Burkitt leukemia and lymphoma. Blood. 2004;104(10):3009-3020.

14. Zelenetz AD, Gordon LI, Abramson JS, Advani RH, Bartlett NL, Caimi PF. NCCN Guidelines Insights: B-Cell Lymphomas, Version 3.2019. J Natl Compr Canc Netw. 2019;17(6):650-661. 